\title{
Time Course of Left Ventricular Pressure-Volume Relationship under Various Enddiastolic Volume
}

\author{
Hiroyuki Suga, M.D.
}

\section{Summary}

A variable, $e(t)=p(t) / v(t)$, is proposed for formulating the ventricular pumping of blood, as a concept of the reciprocal of ventricular compliance, where $t=$ time, $p(t)=$ time course of the intraventricular pressure and $v(t)=$ that of the intraventricular volume. For actual computation, $\mathrm{e}(\mathrm{t})=\mathrm{p}(\mathrm{t}) /\left\{\mathrm{v}(\mathrm{o})-\int_{0}^{\mathrm{t}} \mathrm{i}(\mathrm{t}) \mathrm{dt}\right\}$ where $\mathrm{v}(\mathrm{o})=\mathrm{v}(\mathrm{t})$ at $\mathrm{t}=\mathrm{o}$ or enddiastolic volume and $i(t)=$ aortic blood flow. $v(o)=(1-\rho)^{-1} \cdot v_{s}$, where $\rho=$ residual volume ratio measured by a thermodilution method and $v_{s}=$ stroke volume or integral of $i(t)$ in the cardiac cycle. $e(t)$ is calculated of areflexive left ventricles of dogs under various $v(o)$ controlled by occlusion of the vena cava. In spite of conspicuous changes of $p(t), i(t)$ and $v(t), e(t)$ has approximately unchanged time course and magnitude in a wide range of $\mathrm{v}(\mathrm{o})$. A model of ventricular pumping is presented with $\mathrm{e}(\mathrm{t})$.

\section{Additional Indexing Words :}

Ventricular elastance Ventricular distensibility Ventricular pumping model

$7 \mathrm{HE}$ cardiac ventricle pumps blood from the low pressure into the high pressure system. When the pumping ventricle is simplified as a lumped parameter system, the intraventricular pressure and its volume are considered to be the significant variables of the system. ${ }^{1)}$ The relationship between pressure and volume in a chamber can be described by its compliance $\mathrm{c}: \mathrm{c}=$ volume/ pressure. Since pressure and volume of the ventricle are functions of time, the ventricular compliance or its reciprocal may also be a function of time. If its time course is known, the relationship of pressure and volume of the ventricle is formulated and this would be useful for analysis of ventricular pumping. The purpose of the present investigation is to study whether ventricular pumping is described by the concept of the reciprocal of compliance, under various enddiastolic volume.

From the Institute for Medical Electronics, Faculty of Medicine, University of Tokyo, Tokyo. Received for publication July 10, 1969. 


\section{Mathematical Formulation}

The relationship between pressure and volume of the ventricle is considered to be described by the following equation, based on ordinary pressure-volume diagrams of the ventricle :2)

$$
p(t)=f_{t}\{v(t)\}
$$

where $\mathrm{p}(\mathrm{t})=$ intraventricular pressure, $\mathrm{v}(\mathrm{t})=$ intraventricular volume, $\mathrm{t}=$ time, and $f_{t}$ is a function of time which seems characteristic of the ventricle. Observation of $p(t)$ and $v(t)$ suggests that $f_{t}$ is gradually increasing and then decreasing in the systolic phase. ${ }^{3)}$ A fully-contracted and a fully-relaxed pressure-volume curves show that there is some nonlinearity of $f_{t}$ to $\left.v(t)_{2}{ }^{4}\right)$ but linearity of $f_{t}$ to $v(t)$ is considered to be a good approximation in a wide range of $v(t) .^{5)}$ With the assumption of this linearity, $\mathrm{p}(\mathrm{t})$ and $\mathrm{v}(\mathrm{t})$ are described as follows : $\left.{ }^{6}\right)$

$$
\mathrm{p}(\mathrm{t})=\mathrm{e}(\mathrm{t}) \cdot \mathrm{v}(\mathrm{t})
$$

where $e(t)$ is the concept of the reciprocal of compliance, as a function of time and is gradually increasing and then decreasing in the systolic phase.

\section{MEthods}

$\mathrm{p}(\mathrm{t})$ was measured with an electric manometer. $v(t)$ was measured indirectly by measurements of the aortic blood flow $\mathrm{i}(\mathrm{t})$ with an electromagnetic flowmeter, and of the residual volume ratio $\rho=$ endsystolic volume / enddiastolic volume, with a thermodilution method." The rate of decrease of $v(t)$ was approximated by $i(t)$, because the coronary flow is known to be negligibly small in the systolic phase. ${ }^{8)}$ The equations to be used in the calculation of $\mathrm{v}(\mathrm{t})$ are as follows :

$$
\begin{gathered}
\rho=\sum_{i=1}^{n-1}\left(T_{i+1} / T_{i}\right) /(n-1), \\
v(t)=(1-\rho)^{-1} v_{s}-\int_{0}^{t} i(t) d t
\end{gathered}
$$

where $T_{i}(i=1,2, \ldots, n)=$ difference between baseline aortic temperature and that resulting in the aorta from each systole, as measured at the enddiastole, $\mathrm{n}=$ number of $T_{i}$ 's obtained in a trial, and $v_{s}=$ stroke volume. Only the ratio of $T_{i+1}$ to $T_{i}$ is necessary and the calibration is not required.

Mongrel dogs (12 dogs, 8-12 Kg.) were anesthetized with sodium pentobarbital (25 mg./Kg.). A transverse thoracotomy was done in the 3rd intercostal space under artificial positive pressure respiration. An electromagnetic flowmeter probe was placed at the aortic root as shown in Fig. 1. A catheter with a thermistor (time constant of $50 \mathrm{msec}$.) at its tip was introduced into the aortic root about 1 or $2 \mathrm{~cm}$. above the aortic valve by way of the common carotid artery or internal mammary artery. Another catheter, with its tip occluded and several side holes, was introduced into the left ventricle by way of the left atrial appendage or through the apex. This catheter was used either for measurement of $p(t)$ or for injection of a small 


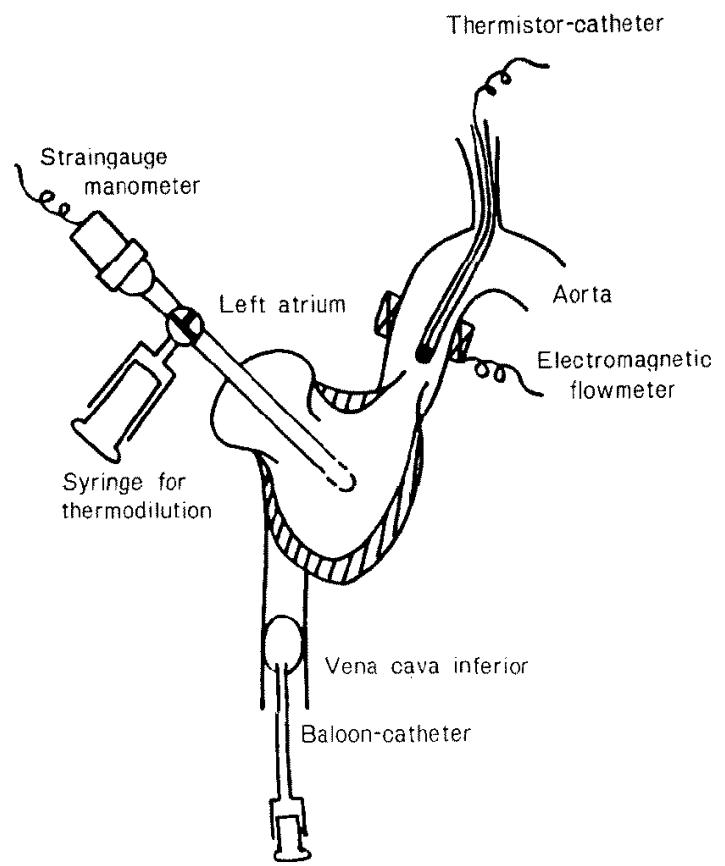

Fig. 1. Schematic diagram of the experiment.

amount of saline (ca. 1-2 ml.) into the left ventricle. Temperature of injected saline was slightly higher or lower than body temperature of the dog, and the amount and the temperature of the injected saline were not necessary to be known. A balloon catheter was introduced into the inferior vena cava by way of the right femoral vein, in order to control venous return and then $v(o)$. Bilateral vagi were severed in the neck. Bilateral stellate ganglia were isolated from their branches except cardiac branches, or in some dogs, beta-blocker (propranorol) was administered i.v. (1 mg./ Kg.). $\quad p(t), i(t)$ and thermodilution were recorded by an ink-pen writer at a speed of 50 or $125 \mathrm{~mm}$. $/ \mathrm{sec}$., and $\mathrm{p}(\mathrm{t})$ and $\mathrm{i}(\mathrm{t})$ were sampled every 10 or $20 \mathrm{msec}$. Measurement of $T_{i}$ was started from the 3rd or 4th enddiastole after completion of saline injection, and ended with the final enddiastole where the temperature difference was accurately readable. The same procedure was repeated 3 or 4 times and their mean was used for calculation of $e(t)$ in one steady condition.

\section{Results}

Fig. 2 is an example of the simultaneous recordings of $i(t), p(t)$ and thermodilution. During injection of saline, $\mathrm{p}(\mathrm{t})$ recording was intermitted. An extrasystole was infrequently observed as a disturbance due to injection, and the case was not used for this study. Fig. 3 is an example of the simultaneous tracings of $\mathrm{i}(\mathrm{t}), \mathrm{p}(\mathrm{t})$ and $\mathrm{EKG}$ under different $\mathrm{v}(\mathrm{o}) . \quad \rho$ and $\mathrm{v}(\mathrm{o})$ are numerically shown in the figure. The amplitudes of $i(t)$ and $p(t)$ changed considerably with 
$\mathrm{v}(\mathrm{o})$, but tendencies of their time course remained almost unchanged. $\rho$ was increased as venous return was decreased. From these data, $\mathrm{e}(\mathrm{t})$ was calculated using equations (3) and (4). The means and the standard deviations of every $20 \mathrm{msec}$. calculated values of $\mathrm{e}(\mathrm{t})$ 's under various $\mathrm{v}(\mathrm{o})$ in a dog were calculated and plotted as in Fig. 4. All e(t)'s of a dog were similar in the time course, except some at low $v(o)$ where $\rho$ 's had more variability than at normal $v(o)$.

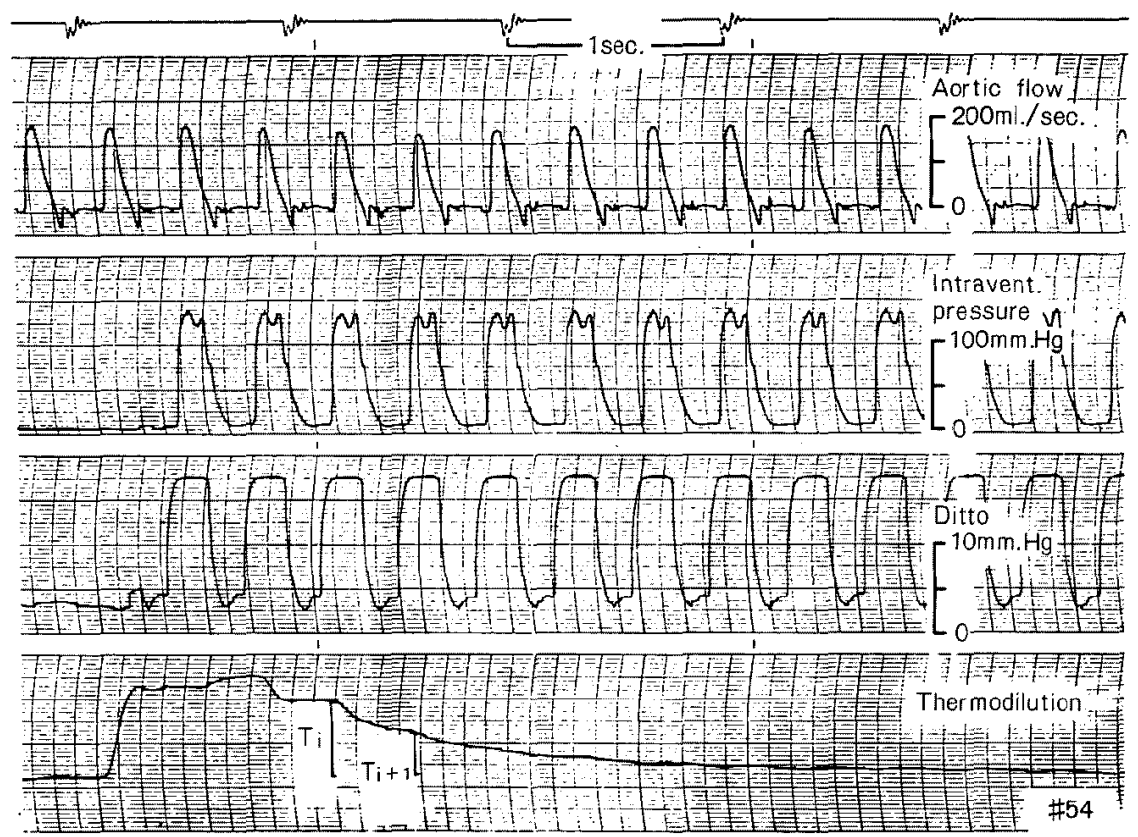

Fig. 2. An example of simultaneous tracings of aortic flow, intraventricular pressure, diastolic pressure and temperature of aortic blood, from above down.

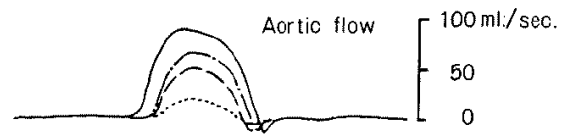

Residual volume ratio (SD)

$$
\begin{array}{lll}
\text { a: } & 0.63 & (0.02) \\
\text { b: } & 0.67 & (0.02) \\
\text { c: } & 0.72 & (0.02) \\
\text { d: } & 0.80 & (0.02)
\end{array}
$$

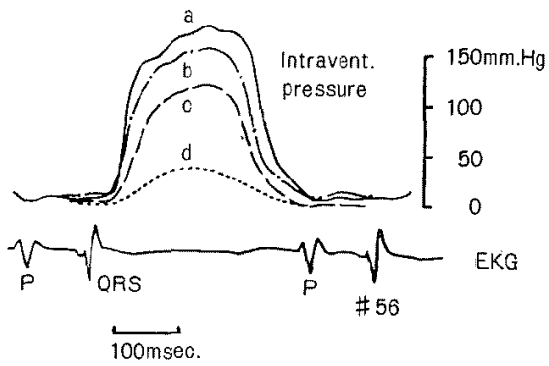

End-diastolic volume $\mathrm{ml}$.
a: 30.8
b: 21.2
c: 16.1
d: 7.8

Fig. 3. Comparison of aortic flow and intraventricular pressure with thermodilution data, under various enddiastolic volume. 
Fig. 5 shows typical e(t)'s of many dogs, normalized to the maximum value of each $e(t)$. There was a tendency that all $e(t)$ 's were alike in their time courses: the rising gradient consisted of two portions, the initial portion being steeper; the peak was in the latter half of the systolic phase; the maximum falling gradient was steeper than that of the rising gradient.

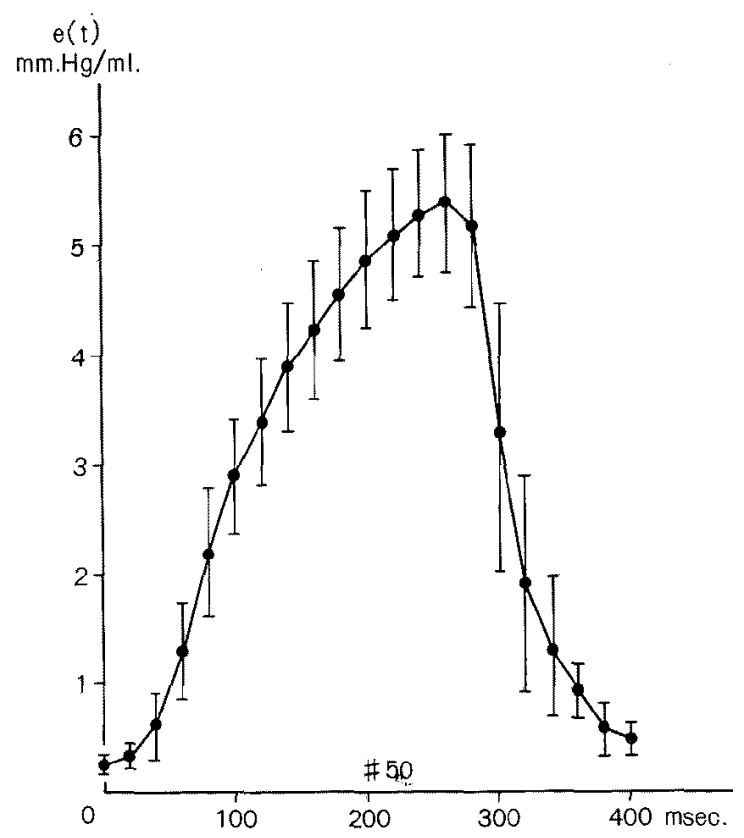

Fig. 4. Means and standard deviations of $e(t)$ 's in a wide range of enddiastoliclvolume. Closed circle: mean, and I : standard deviation.

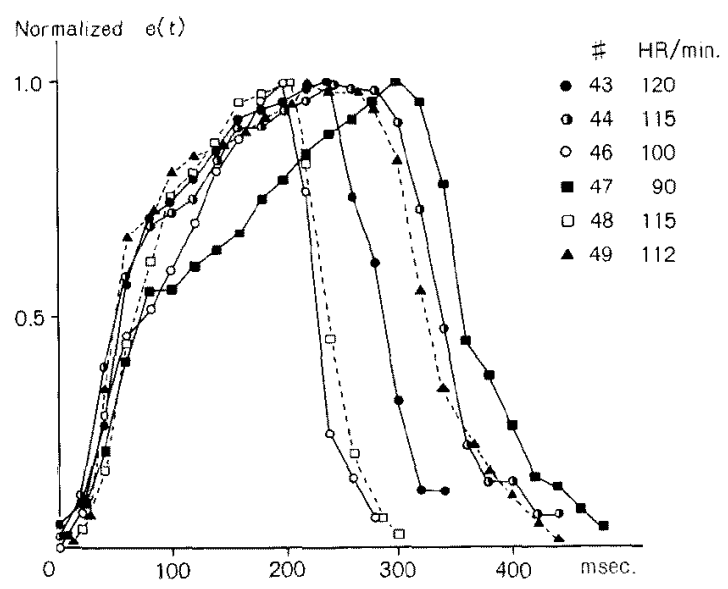

Fig. 5. Comparison of $e(t)$ 's of several dogs with vagotomy and stellectomy. Each $\mathrm{e}(\mathrm{t})$ is normalized to its own maximum. 


\section{Discussion}

The most indirectly measured variable in computation of $e(t)$ is $\rho$ by the thermodilution method, which is valid only when the ventricle is a complete mixer of heat. Variability of $\rho$ measured by an indicator dilution method is known to be due to the incomplete mixing in the left ventricle. ${ }^{9)}$ To eliminate the variability, the mean of several trials is used in computation of $\left.e(t) .{ }^{9}\right)$ Standard deviation of $\rho$ is at most 0.05 and usually 0.02 in this investigation. Since $\rho$ is usually between 0.4 and 0.8 , standard deviation of $\rho$ of 0.05 is between 10 and $25 \%$ of $1-\rho$, and that of 0.02 is between 3 and $5 \%$ of $1-\rho$, hence the accuracy of $\mathrm{v}(\mathrm{o})$ and $\mathrm{e}(\mathrm{t})$ is decreased significantly by the variability of $\rho$.

In this paper, independency of $e(t)$ from $v(t)$ is investigated. With some variability, $\mathrm{p}(\mathrm{t})$ at maximum $\mathrm{e}(\mathrm{t})$ in a pressure-volume chart increases approximately proportionally to increase of $\mathrm{v}(\mathrm{t})$, and in most dogs the maximum $\mathrm{e}(\mathrm{t})$ and its time in an individual is approximately constant. The position of the maximum $e(t)$ in the cardiac phase is posterior to the peaks of $i(t)$ and $p(t)$, and anterior to the closing time of the aortic valve. $p(t)$ at the maximum $e(t)$ is different from the peak systolic pressure in auxotonic contraction, but is the upper-leftmost positioned pressure in a pressure-volume diagram.

In spite of the variability of $e(t)$, almost all the calculated $e(t)$ 's have the same tendency of time course, with remarkable changes of other hemodynamic variables. The tendency of $e(t)$ of the areflexive hearts in this investigation is similar to that of hearts with cardiac nerves intact. ${ }^{10}$ Stimulation of cardiac sympathetic nerves, vagus nerves, or heart rate change does not change the tendency of time course of $\mathrm{e}(\mathrm{t})$, in spite that sympathetic stimulation increases the magnitude of $e(t)$ and shortens the systolic duration. $\left.{ }^{6}\right) \quad$ These experimental results suggest that $e(t)$ might be a stable and characteristic parameter of the left ventricle.

From these discussions, a block diagram of the ventricular pumping model

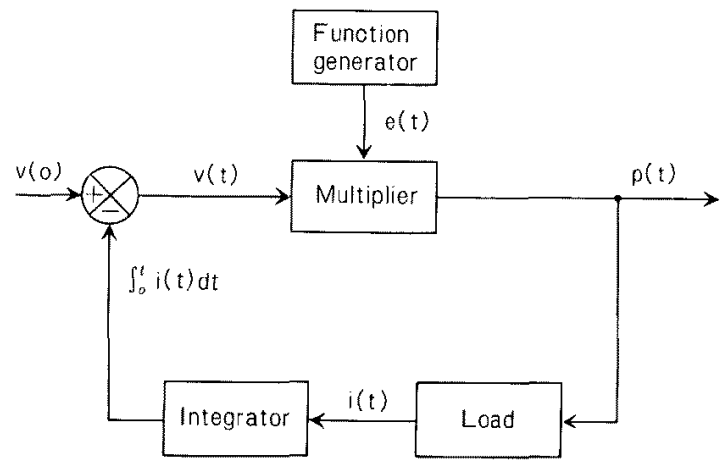

Fig. 6. Block diagram of ventricular pumping model. 
is shown in Fig. 6. $v(0)$ is considered to be determined by the venous return independently from $p(t)$ and $i(t)$. $e(t)$ is the above-mentioned function, characteristic of the ventricle. The load is the arterial and venous system distal from the aortic root, and characterized by the hydraulic impedance. The multiplier and the integrator compute $\mathrm{e}(\mathrm{t}) \cdot \mathrm{v}(\mathrm{t})$ and $\int_{0}^{t} \mathrm{i}(\mathrm{t}) \mathrm{dt}$, respectively. The function generator generates $e(t)$ periodically and its period is equivalent to heart beat interval. At the beginning of each cycle, $t$ is zero when integration is set to zero. Analysis of this model has been under investigation, and some examples are published, ${ }^{6}$ which show that this model, with some assumptions of $e(t)$ and the characteristics of the load, simulates relationships among many hemodynamic variables.

\section{AGKNOWLeDGEMENT}

The author is indebted to Prof. Masamitsu Oshima, M.D. for the continuing guidance.

\section{REFERENGES}

1. Frank, O.: Zeitschrift für Biologic 32:370, 1895 (Transl. by Chapman, C. B. and Wasserman, E.: Am. Heart J. 58: 282 and 467, 1959).

2. Straub, H.: Pflüg. Arch. 169:564, 1917.

3. Henderson, Y.: Am. J. Physiol. 16 : 325, 1906.

4. Ullrich, K.J., Riecker, G., and Kramer, K.: Pfüg. Arch. 259 ; 481, 1954.

5. Monroe, G. and French, G. N. : Circulat. Res. 9: 362, 1961.

6. Suga, H.: MBE 69-6 (1969-07), The Institute of Electronics and Communication Engineers of Japan, 1969.

7. Holt, J. P.: Circulat. Res. 4: 187, 1956.

8. Gregg, D. E. and Fisher, L. C.: Handbook of Physiology; Circulation, Vol. 2 : 1517, Am. Physiol. Soc., 1963.

9. Irisawa, H., Wilson, M. F., and Rushmer, R. F.: Circulat. Res. 8: 183, 1960.

10. Suga, H.: J. Physiol. Soc. Japan 31: 328, 1969. 